\title{
Entrapped Urinary Calculi in Iatrogenically Created Vaginal Pocket During Robotic Repair of Vesicovaginal Fistula
}

\author{
Zebulun Cope $^{\mathbf{1}^{*}}$, Raisa Platte ${ }^{1}$
}

\begin{abstract}
Primary vaginal calculi exist as an extremely rare topic discussed only through a handful of case reports. Based on the cases studied, it was reported that they have an association with urethral diverticulum, urogenital sinus anomalies, urogenital fistula or with utilization of transvaginal urethral slings. We present a case of an incidental finding of multiple vaginal calculi impacted in the proximal vagina in an iatrogenically created pocket after a failed Latzko procedure which were later discovered during a robotically assisted laparoscopic vesicovaginal fistula (VVF) repair by the extravesical approach. This is the first reported incident of multiple vaginal calculi associated with iatrogenically created intravaginal pocket after Latzko procedure.

Keywords: Calculi, Robotic surgery, Vesicovaginal Fistula
\end{abstract}

\section{Introduction}

Vaginal calculi are rare and when they occur, they are often associated with a urogenital anomaly, urethral diverticulum, urinary tract fistulas or as a complication encountered with synthetic transvaginal mid-urethral slings. Vaginal calculi are described as either primary or secondary. A primary vaginal calculus is the result of stagnant urine within the vagina, while a secondary calculus is the product of crystalized urine around foreign material within the vagina. This case report presents the first description of a primary vaginal calculus in an iatrogenically created pocket after a failed Latzko procedure.

\section{Case Presentation}

The patient was a 43-year-old multiparous woman who originally presented to our office two months after an uncomplicated total abdominal hysterectomy (TAH) with the chief concern of continuous urinary incontinence occurring since that surgery. Her past medical history was significant for obesity, cervical intraepithelial neoplasia II (CIN II) and 3 prior caesarean deliveries. A review of her family history proved significant for malignant hyperthermia.

During the initial physical exam, the patient presented with an unremarkable appearance of the vulva and a well-supported perineum with adequate apical support. A retrograde voiding cystogram confirmed the presence of a vesicovaginal fistula (VVF). Visual identification of the fistulous opening proved to be challenging. After an unsuccessful exam in the clinic, the decision was made to perform an exam under anesthesia (EUA) in the operating room to identify the fistula and determine the optimal route for repair.

EUA revealed a small area of granulation tissue within the vagina with concomitant spillage of indigo carmine that had been instilled within the bladder. Cystourethroscopy showed an unremarkable appearing urethra and bladder with bilateral orthotropic ureters freely effluxing indigocarmine colored urine. A $1-\mathrm{cm}$ fistula, $2 \mathrm{~cm}$ medial to the left ureter and $2 \mathrm{~cm}$ superior to the trigone, was visualized. A 6-French pediatric catheter was then utilized to tubularize the fistula. Repeat cystourethroscopy confirmed placement of the catheter through the fistulous tract. Placing gentle traction on the catheter brought the vaginal cuff to mid-position within the vagina allowing a Latzko procedure with its vaginal approach feasible.

Following the exposure to the vaginal apex, a fullthickness, circumferential dissection of the vaginal wall was performed to expose the fistulous tract by creating tension free vaginal flaps for future closure of the fistula. The fistula was then isolated and closed in 2 layers first by using 2-0 Vicryl on the vaginal side of the fistula followed by the creation of an imbricating layer. The closure of the fistula was tested for water-tightness by instilling 350 $\mathrm{mL}$ of diluted methylene blue in normal saline into the bladder which revealed the absence of any leakage. The vaginal cuff was then reapproximated for closure with 3-0 Vicryl and the cystoscopy that followed confirmed absence of sutures within the bladder urothelium. Her 
postoperative recovery was complicated by an inability to tolerate an indwelling urinary catheter in the immediate days after the operation. She removed the catheter on postoperative day 5 due to discomfort despite having an antispasmodic bladder regimen of phenazopyridine PO $200 \mathrm{mg}$ TID and Oxybutynin PO $5 \mathrm{mg}$ TID. Two weeks after the Latzko procedure, a urine analysis showed no laboratory evidence of a urinary tract infection, however, a cystogram revealed the persistence of the VVF.

Due to the recurrence of the VVF, a Da Vinci robot assisted laparoscopic extravesical repair with omental intraposition graft was proposed to repair the persistent VVF. The surgery was delayed by more than six months due to the patient's work schedule and time commitment to her children and family.

We eventually were able to schedule a surgery date that was optimal for the patient considering her schedule. During the day of surgery, once back in the operating room, an EUA revealed a surgically absent cervix and uterus, no adnexal masses and an unexpected firm nodularity at the apex of the vagina. The large nodular mass was later confirmed to be multiple urinary stones impacted within an iatrogenically created pocket at the proximal vagina via dissection (Figures 1-3). Those stones upon further analysis were found to be $50 \%$ calcium phosphate (apatite) and $40 \%$ magnesium ammonium phosphate (struvite).

The location of the stone was postulated to be related to the previous Latzko procedure which had created an ideal reservoir for urine entrapment. Sedimentation of the urine then took place resulting in the formation of urinary stones.

The patient did well after the surgery. She was able to be compliant with the indwelling Foley catheter during her postoperative period this time. Her cystourethrogram after 2 weeks was consistent with an intact urinary bladder without evidence of a fistula. She continued to be continent at 6 and 12 months postoperatively.

\section{Conclusions}

The presence of vaginal calculi has been well established (1-4). However, they remain very rare in occurrence in the medical literature. When these anomalous stones occur, they often develop in conjunction with a VVF, urethrovaginal fistula or an implanted material such as a mesh containing device. The precedence in this case then is not the appearance of the vaginal stones, but rather their creation through an iatrogenically produced VVF repair and their subsequent seeding via that route.

An interesting point in this case is that during the patient's first repair of the fistula using a vaginal approach (Latzko procedure), neither the EUA nor the surgery itself revealed calculi of any kind. The implication then is that the prior attempted repair served as a seeding of necessary elements within the proximal vagina to promote the formation of the vaginal calculi between the vaginal layers during the Latzko procedure. Immediately, on EUA

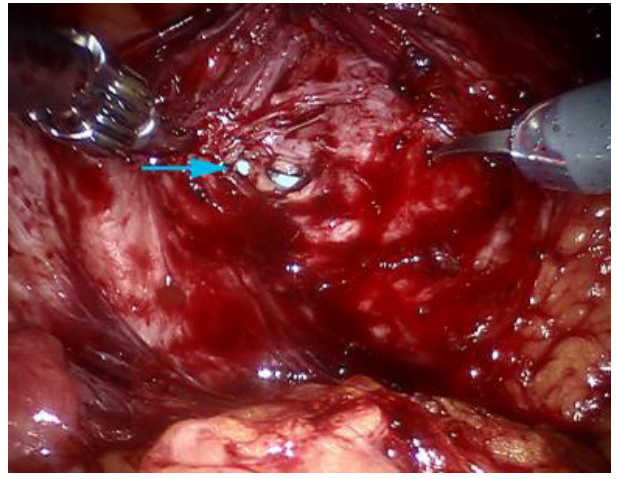

Figure 1. Emergence of the catheter (blue arrow), visualized after dissection, placed through fistulous tract with the bladder to the left and superiorly and apex of the vagina to the right and inferiorly.

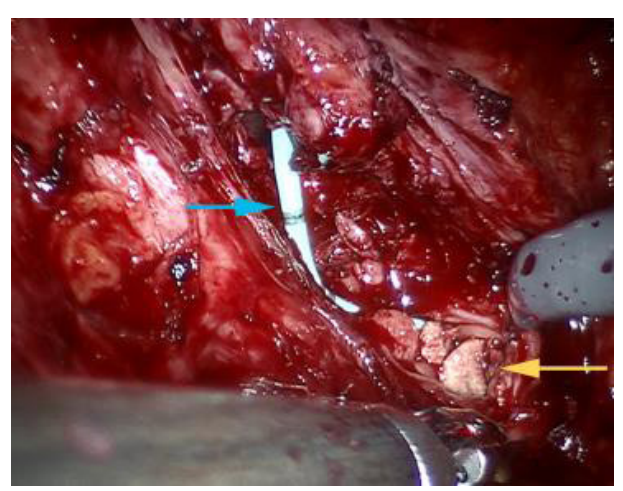

Figure 2. Passage of catheter (blue arrow) through the fistulous opening in the bladder, seen superiorly, into the apex of the vagina with emerging calculi (gold arrow) visualized after dissection.

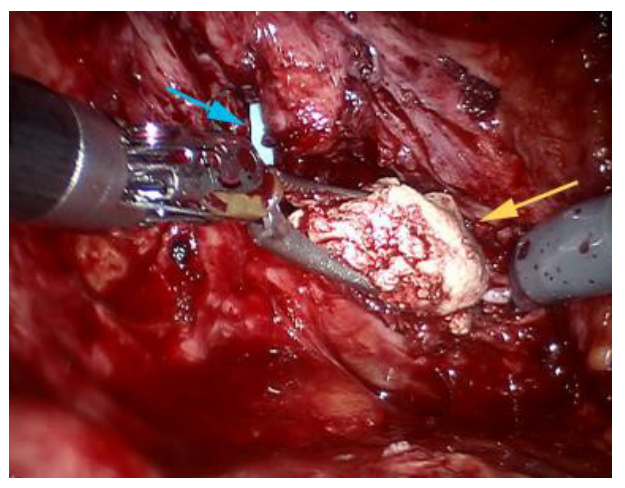

Figure 3. Catheter (blue arrow) passing through fistulous tract with bladder superiorly and the apex of vagina inferiorly with the presence of vaginal calculi (gold arrow) visualized after continued dissection.

during the second procedure to repair the VVF, a firm mass could be palpated in the apical portion of the vagina which would later be discovered to be calculi.

In regards to the seeding of the stones, a urine culture had been performed secondary to a urinary tract infection reported in the patient the week prior to the final fistula repair. The results showed greater than 100,000 colony forming units of both Pseudomonas aeruginosa and 
Klebsiella oxytoca. An association between the presence of bacteria belonging to the Enterobacteriaceae family, to which $K$. oxytoca belongs, and the presence of calcium oxalate stones has been discussed in the literature (5). Speaking of the stones, $40 \%$ struvite composition, urease producing bacteria, to which $P$. aeruginosa belongs, are well known to be a component of struvite formation.

We postulate that the presence of the vaginal calculi was the result of an iatrogenically produced fistula that led to the first attempted repair that then permitted seeding of the necessary elements to produce the stones due to failure to reapproximate multiple vaginal layers adequately. The stasis of the bacteria-ridden urine within the separated vaginal layers created an ideal environment for stone formation. The presence of calculi is not an arbitrary point. As it stands, an element that we are unable to determine is if, in fact, the formation of the calculi contributed to the persistence of the fistula after the attempted Latzko repair.

\section{Conflict of Interests}

The authors declare that they have no conflicts of interest and nothing to disclose.

\section{Ethical Issues}

IRB approval was not required for this case report as no patient identifiers have been used.

\section{Financial Support}

None.

\section{References}

1. Ranawaka RS, Goyal A, Shabani A, Hennayake S, Dickson AP, Cervellione RM. Novel approach to vaginal calculus in a girl with urogenital sinus anomaly. J Pediatr Surg. 2015. doi:10.1016/j.jpedsurg.2012.07.033

2. Tavakkoli M, Ghoreifi A. Large vaginal and bladder calculi in a woman with previous operation of bladder exstrophy: a case report. Nephrourol Mon. 2014;6(1):e13637. doi:10.5812/numonthly.13637

3. Zilberlicht A, Feiner B, Haya N, Auslender R, Abramov Y. Surgical removal of a large vaginal calculus formed after a tension-free vaginal tape procedure. Int Urogynecol J. 2016;27(11):1771-1772. doi:10.1007/s00192-016-3065-x

4. Raghavaiah NV, Devi AI. Primary vaginal stones. J Urol. 1980;123(5):771-772.

5. Barr-Beare E, Saxena V, Hilt EE, et al. The Interaction between Enterobacteriaceae and Calcium Oxalate Deposits. PLoS One. 2015;10(10):e0139575. doi:10.1371/journal. pone. 0139575

(C) 2019 The Author (s); This is an open-access article distributed under the terms of the Creative Commons Attribution License (http://creativecommons.org/licenses/by/4.0), which permits unrestricted use, distribution, and reproduction in any medium, provided the original work is properly cited. 